\title{
Measuring the Masses: Understanding Health Outcomes Arising from Mass Gatherings, Reporting Gaps, and Recommendations (Paper 2)
}

\author{
Sheila Turris, RN, BHSc, MSN, PhD; ${ }^{1,2}$ Haddon Rabb, BSc, RN; ${ }^{2} \odot$ Matthew Brendan Munn, MD, \\ MPhil, CCFP(EM-FPA), DA(SA); ${ }^{1,2}$ Elizabeth Chasmar, BASc; ${ }^{2}$ Christopher W. Callaghan, BSc; ${ }^{2}$ \\ Jamie Ranse, RN, PhD; ${ }^{3,4}$ Adam Lund, BSc, MD, MEd, FRCPC (Emergency) ${ }^{1,2,5}$
}

1. Department of Emergency Medicine, University of British Columbia, Vancouver, British Columbia, Canada

2. Mass Gathering Medicine Interest Group, Department of Emergency Medicine, University of British Columbia, Canada

3. Department of Emergency Medicine, Gold Coast Health, Southport, Queensland, Australia

4. Menzies Health Institute Queensland, Griffith University, Gold Coast, Queensland, Australia

5. School of Nursing, University of British Columbia, Vancouver, British Columbia, Canada

Correspondence:

Haddon Rabb, BSc, RN

Mass Gathering Medicine Interest Group

Department of Emergency Medicine

University of British Columbia

Vancouver, British Columbia, Canada

E-mail: haddonrabb@gmail.com

Conflicts of interest/funding: Turris is a shareholder with a medical services company that provides health care services for mass gatherings. Rabb, Chasmar, Ranse, and Callaghan have no Conflicts of Interest to declare. Lund is the medical director and a shareholder of a medical services company that provides health care services for mass gatherings. All the authors take on both volunteer and paid roles providing medical services at mass gatherings. None of the authors received income for this project, which is unfunded.

Keywords: mass gathering; mass-gathering health; mass-gathering medicine

Abbreviations:

COVID-19: coronavirus disease 2019

MG: mass gathering
Turris S, Rabb H, Munn MB, Chasmar E, Callaghan CW, Ranse J, Lund A. Measuring the masses: understanding health outcomes arising from mass gatherings, reporting gaps, and recommendations (paper 2). Prehosp Disaster Med. 2021;36(2):243-245.

Within the world of mass-gathering (MG) medicine and event medicine, researchers are making continued efforts to standardize data collection, analysis, and reporting. ${ }^{1-6}$ Given the number of MGs that occur annually around the globe, and the associated mortality and morbidity that can arise, it is time to harmonize post-event reporting on health outcomes. In the context of the present coronavirus disease 2019 (COVID-19) pandemic, there is a world-wide "pause" on MGs. Perhaps this is the time for the MG research community to realign and sharpen the focus of this evolving academic field. Coordinating post-event reporting through the consistent capture and publication of essential data points will allow researchers and clinicians to make comparisons between events and across event types with the goal of mitigating or obviating negative outcomes for individuals and communities.

In a recent paper, Turris, et al carried out an analysis of the current state of post-event reporting, with a focus on understanding and documenting patterns. The study found widespread reporting inconsistencies. ${ }^{7}$ As expected, a number of recommendations arise from an analysis of current state. Below is a summary of six identified gaps in reporting, along with ten initial recommendations for beginning to address the gaps (Table 1). These recommendations lay out a beginning roadmap for subsequent papers that may assist in addressing these important issues in the literature.

\section{Language \& Terminology}

Gap analysis: There is currently no guideline or template supporting consistent labelling of MG medical case reports; inconsistent labeling creates issues related to manuscript searchability, accessibility, and comparability.

1. Standardize the keywords used in post-event MG case reporting. The authors propose that (at minimum) the following keywords be listed for every case report: "mass-gathering medicine," "case report/case series," and a word or phrase that describes the event type (eg, marathon, music festival).

2. Standardize the format of titles. The authors propose that the phrase "case report" or "case series" and the type of event should appear in the title (eg, "Case Report on an Obstacle Adventure Course in a Hot Climate" or "Patient Outcomes from a MultiDay Music Festival in a Hot Climate: A Case Report").

Received: October 12, 2020

Accepted: November 17, 2020

doi:10.1017/S1049023X21000078

(C) The Author(s), 2021. Published by Cambridge University Press on behalf of the World Association for Disaster and Emergency Medicine. 


\begin{tabular}{|c|c|}
\hline $\begin{array}{l}\text { Recommendation } \\
\text { Number }\end{array}$ & Case Reporting Recommendation \\
\hline 1 & $\begin{array}{l}\text { Standardize the keywords used in post-event mass gathering case reporting. The authors propose that (at minimum) the } \\
\text { following keywords be listed for every case report: "mass gathering medicine," "case report/case series," and a word or } \\
\text { phrase that describes the event type (eg, marathon, music festival). }\end{array}$ \\
\hline 2 & $\begin{array}{l}\text { Standardize the format of titles. The authors propose that the phrase "case report" or "case series" and the type of event } \\
\text { should appear in the title (eg, "Case Report on an Obstacle Adventure Course in a Hot Climate" or "Patient Outcomes from a } \\
\text { Multi-Day Music Festival in a Hot Climate: A Case Report"). }\end{array}$ \\
\hline 4 & $\begin{array}{l}\text { Work toward standardizing the language, where possible, and embedding agreed upon terms into an event reporting } \\
\text { template that can evolve over time as the understanding of what constitutes essential data points grows. }\end{array}$ \\
\hline 5 & $\begin{array}{l}\text { When reporting quantitative data (eg, temperature, area, distances, and crowd density), the values should be reported in } \\
\text { accordance to the metric system. }\end{array}$ \\
\hline 6 & $\begin{array}{l}\text { Develop and evolve quantitative and qualitative data models to provide guidance around what constitutes essential core } \\
\text { data points for reporting post-event health outcomes, including categories of data currently inconsistently reported (eg, } \\
\text { community capacity, scope of practice for on-site health care teams). }\end{array}$ \\
\hline 8 & $\begin{array}{l}\text { Standardize reporting on the composition of the on-site first aid, health, and emergency services, which will inform future } \\
\text { efforts to more accurately predict and match medical services to needs for specific types and sizes of mass gatherings. }\end{array}$ \\
\hline 9 & $\begin{array}{l}\text { Standardize the ways in which illnesses and injuries are categorized and reported through the development of qualitative } \\
\text { descriptors and a quantitative data model (see above) that would provide guidance for researchers and clinicians around } \\
\text { essential data points. }\end{array}$ \\
\hline 10 & $\begin{array}{l}\text { Researchers should report essential, event-related statistics in a standardized way. For example, the source of the } \\
\text { attendance number being provided, including metrics that do not require an accurate denominator of overall attendance. }\end{array}$ \\
\hline
\end{tabular}

Table 1. Mass Gathering Post-Event Case Reporting Recommendations

Gap analysis: There is currently no guideline or template that supports/provides consensus on terminology; inconsistent use of terminology creates issues related to both retrieval and comparison of data points.

3. Future research efforts should focus on developing and evolving a typology that would standardize event naming and categorization or tagging to improve the ease of comparisons between similar and dissimilar events.

4. Work toward standardizing the language, where possible, and embedding agreed upon terms into an event reporting template that can evolve over time as the understanding of what constitutes essential data points grows.

5. When reporting quantitative data (eg, temperature, length, area, distances, and crowd density), the values should be reported in accordance to the metric system.

\section{Data Modelling}

Gap analysis: There is currently no robust conceptual model that might drive analysis and understanding of event-specific data. As a result, reports are sometimes limited to convenient data points, which are often insufficient to provide a deep understanding how factors related to a specific event might be interacting to influence health outcomes.

6. Develop and evolve quantitative and qualitative data models to provide guidance around what constitutes essential core data points for reporting post-event health outcomes, including categories of data currently inconsistently reported (eg, community capacity, scope of practice for on-site health care teams).
Hazard \& Risk Analysis

Gap analysis: There is currently no guideline or template for structured reporting on hazards, risks, and risk mitigation in the context of post-event medical case reports.

7. The authors propose that hazards and risks be explored and explained, providing information on hazards and risks per event type AND per specific event with a future goal of utilizing these discussions to inform the development of an efficient and user-friendly set of event-specific hazard and risk-assessment tools.

\section{Capacity Analysis}

Gap Analysis: There is currently no guideline or template for reporting on the composition of on-site medical teams or determining the degree of fit between the size/composition of the team and the actual need per event. In addition, there is currently no approach to quantifying and describing public health interventions (ie, "non-medical" services/strategies that may improve health outcomes and mitigate risk for attendees and participants at MGs).

8. Standardize reporting on the composition of the on-site first aid, health, and emergency services, which will inform future efforts to more accurately predict and match medical services to needs for specific types and sizes of MGs.

\section{Health Outcomes Measurement \& Reporting}

Gap analysis: There is currently no guideline or template for consistent analysis and reporting of patient-related data/outcomes. 
9. Standardize the ways in which illnesses and injuries are categorized and reported through the development of qualitative descriptors and a quantitative data model (see above) that would provide guidance for researchers and clinicians around essential data points.

10. Researchers should report essential, event-related statistics in a standardized way. For example, the source of the attendance number being provided, including metrics that do not require an accurate denominator of overall attendance.

\section{Conclusion}

Humanity is living through a pandemic that has presented a multitude of challenges that have been, and continue to be, faced by countries around the globe. One of the first efforts to "flatten the curve" of COVID-19 infection rates involved social/physical distancing and so all MGs were cancelled or postponed. A consistent area of focus has been the collection and analysis of data so that everyone can understand what is actually happening in terms of measurable, relevant outcomes. Taking a page from the excellent work being done by COVID-19 researchers, it is time to focus international attention more fully on improving data quality vis a vis MGs and health outcomes.

In submitting this letter, and the accompanying publications that include qualitative and quantitative data models, and a proposed version of a reporting template, the authors invite engagement and debate with the goal of improving data modelling and reporting of health outcomes related to MGs.

\section{References}

1. Arbon P. The development of conceptual models for mass-gathering health. Prehosp Disaster Med. 2004;19(3):208-212.

2. Schwellnus M, Kipps C, Roberts WO, et al. Medical encounters (including injury and illness) at mass community-based endurance sports events: an international consensus statement on definitions and methods of data recording and reporting. Br J Sports Med. 2019;53(17):1048-1055.

3. Lund A, Turris SA, Bowles R, et al. Mass-gathering health research foundational theory: part 1 - population models for mass gatherings. Prehosp Disaster Med. 2014;29(6):648-654.

4. Hutton A, Ranse J, Gray KL, Turris SA, Lund A, Munn MB. Environmental influences on patient presentations: considerations for research and evaluation at mass-gathering events. Prehosp Disaster Med. 2019;34(05):552-556.

5. Turris SA, Lund A, Hutton A, et al. Mass-gathering health research foundational theory: part 2 - event modeling for mass gatherings. Prehosp Disaster Med. 2014; 29(6):655-663.

6. Hutton A, Ranse J, Gray KL, Turris S, Lund A, Munn MB. Psychosocial influences on patient presentations: considerations for research and evaluation at mass-gathering events. Prehosp Disaster Med. 2020;35(2):197-205.

7. Turris S, Rabb H, Munn MB, et al. Measuring the masses: the current state of mass gathering medical case reporting (paper 1). Prehosp Disaster Med. 2021. In press. 